\title{
Société suisse de génétique médicale (SSGM)
}

Isabel Filges et Peter Miny, pour le comité

Correspondance:

Katharina Neves Secrétariat de la Société suisse de génétique médicale c/o UKBB

CH-4005 Bâle

Tél. 0616856432

Fax 0616856011

Katharina.Neves@ukbb.ch

www.sgmg.ch

www.eurogentest.org

www.orphanet.org

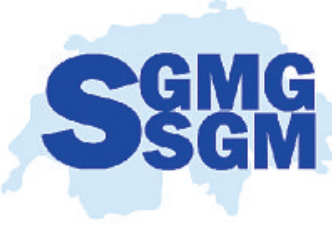

La SSGM a pour objectif de promouvoir la pratique, l'enseignement et la recherche en génétique médicale, de fixer des standards éle-

vés pour le conseil et le diagnostic génétiques et d'assurer une prise en charge appropriée aux personnes souffrant de maladies génétiques en Suisse. Elle constitue une société faîtière qui regroupe les médecins spécialistes FMH en génétique médicale et les spécialistes FAMH en analyse génétique médicale, souvent diplômés en sciences naturelles. Elle se conçoit essentiellement comme la représentante d'une discipline toujours plus sollicitée par de nombreuses autres spécialités médicales et qui ne peut donc remplir sa mission qu'en collaboration étroite avec ces dernières.

\section{Histoire de la SSGM}

La SSGM est une jeune société créée en 1978. La qualification FMH a été formellement instaurée en 1999 et la spécialisation FAMH introduite en 2000. La SSGM travaille aujourd'hui à une adaptation des programmes établis de formation postgraduée, notamment dans le but d'assurer une reconnaissance sans complications des formations spécialisées acquises à l'étranger par de jeunes médecins et naturalistes suisses et de faciliter les échanges internationaux. Le «Human Genome Project» et ses projets successeurs ont fait progresser à pas de géant nos connaissances sur le rôle des gènes dans l'étiologie des maladies au cours des 20 dernières années. Il en est résulté de nouvelles possibilités de diagnostic génétique en laboratoire, pour lesquelles la Suisse a été l'un des premiers pays à se doter d'une législation exemplaire dès 2007 avec la loi fédérale sur l'analyse génétique humaine (LAGH) et son ordonnance (OAGH).

\section{Priorités actuelles}

Collaborant étroitement avec l'administration de la santé et les assureurs-maladie, la SSGM est intervenue pour que les maladies héréditaires monogéniques rares (maladies dites «orphelines») soient prises en compte dans la LAMal, ce qui était souvent une impossibilité formelle et administrative par le passé. Notre autre revendication importante est l'intégration judicieuse de l'analyse génétique chez les personnes parentes de patients porteurs de mutations, par exemple à titre de mesure préventive dans le cadre de la LAMal.

La SSGM soutient résolument tous les efforts en vue de réviser la liste des infirmités congénitales de l'assurance-invalidité (AI) et de l'adapter aux changements intervenus dans l'état des connaissances. Maintenant que les maladies orphelines se sont hissées au rang des priorités d'action des pays de l'UE, il est temps que la Suisse procède aussi à une réévaluation de la situation et fasse cesser les inégalités de traitement actuelles. La base légale se trouve entre autres dans l'art. 4 LAGH, qui interdit toute discrimination fondée sur le patrimoine génétique.

Dans le cadre des révisions prévues du TARMED, la SSGM vise par ailleurs la création d'une valeur intrinsèque en médecine génétique, inexistante à ce jour.

\section{Défis futurs}

L'intégration tant espérée de la médecine génomique au cabinet et à l'hôpital posera un immense défi aux médecins, à l'administration de la santé et aux assureurs-maladie. L'accroissement constant des connaissances sur les composantes génétiques de maladies multifactorielles fréquentes mettra à rude épreuve les mécanismes régulateurs en place. La SSGM considère ici comme sa mission de faire valoir son expertise dans l'évaluation factuelle (evidence-based) des nouvelles méthodes d'analyse et leur introduction dans le diagnostic clinique. 